\title{
CONCENTRATION OF THE INTRINSIC VOLUMES OF A CONVEX BODY
}

\author{
MARTIN LOTZ, MICHAEL B. MCCOY, IVAN NOURDIN, GIOVANNI PECCATI, AND JOEL A. TROPP
}

\begin{abstract}
The intrinsic volumes are measures of the content of a convex body. This paper applies probabilistic and information-theoretic methods to study the sequence of intrinsic volumes. The main result states that the intrinsic volume sequence concentrates sharply around a specific index, called the central intrinsic volume. Furthermore, among all convex bodies whose central intrinsic volume is fixed, an appropriately scaled cube has the intrinsic volume sequence with maximum entropy.
\end{abstract}

\section{Introduction and Main Results}

Intrinsic volumes are the fundamental measures of content for a convex body. Some of the most celebrated results in convex geometry describe the properties of the intrinsic volumes and their interrelationships. In this paper, we identify several new properties of the sequence of intrinsic volumes by exploiting recent results from information theory and geometric functional analysis. In particular, we establish that the mass of the intrinsic volume sequence concentrates sharply around a specific index, which we call the central intrinsic volume. We also demonstrate that a scaled cube has the maximum-entropy distribution of intrinsic volumes among all convex bodies with a fixed central intrinsic volume.

1.1. Convex Bodies and Volume. For each natural number $m$, the Euclidean space $\mathbb{R}^{m}$ is equipped with the $\ell_{2}$ norm $\|\cdot\|$, the associated inner product, and the canonical orthonormal basis. The origin of $\mathbb{R}^{m}$ is written as $\mathbf{0}_{m}$.

Throughout the paper, $n$ denotes a fixed natural number. A convex body in $\mathbb{R}^{n}$ is a compact and convex subset, possibly empty. Throughout this paper, $\mathrm{K}$ will denote a nonempty convex body in $\mathbb{R}^{n}$. The dimension of the convex body, $\operatorname{dim} \mathrm{K}$, is the dimension of the affine hull of $\mathrm{K}$; the dimension takes values in the range $\{0,1,2, \ldots, n\}$. When $\mathrm{K}$ has dimension $j$, we define the $j$-dimensional volume $\mathrm{Vol}_{j}(\mathrm{~K})$ to be the Lebesgue measure of $\mathrm{K}$, computed relative to its affine hull. If $\mathrm{K}$ is 0 -dimensional (i.e., a single point), then $\mathrm{Vol}_{0}(\mathrm{~K})=1$.

For sets $C \subset \mathbb{R}^{n}$ and $\mathrm{D} \subset \mathbb{R}^{m}$, we define the orthogonal direct product

$$
\mathrm{C} \times \mathrm{D}:=\left\{(\boldsymbol{x}, \boldsymbol{y}) \in \mathbb{R}^{n+m}: \boldsymbol{x} \in \mathrm{C} \text { and } \boldsymbol{y} \in \mathrm{D}\right\} .
$$

To be precise, the concatenation $(\boldsymbol{x}, \boldsymbol{y}) \in \mathbb{R}^{n+m}$ places $\boldsymbol{x} \in \mathbb{R}^{n}$ in the first $n$ coordinates and $\boldsymbol{y} \in \mathbb{R}^{m}$ in the remaining $(n-m)$ coordinates. In particular, $\mathrm{K} \times\left\{\mathbf{0}_{m}\right\}$ is the natural embedding of $\mathrm{K}$ into $\mathbb{R}^{n+m}$

Several convex bodies merit special notation. The unit-volume cube is the set $Q_{n}:=[0,1]^{n} \subset \mathbb{R}^{n}$. We write $\mathrm{B}_{n}:=\left\{\boldsymbol{x} \in \mathbb{R}^{n}:\|\boldsymbol{x}\| \leq 1\right\}$ for the Euclidean unit ball. The volume $\kappa_{n}$ and the surface area $\omega_{n}$ of the Euclidean ball are given by the formulas

$$
\kappa_{n}:=\operatorname{Vol}_{n}\left(\mathrm{~B}_{n}\right)=\frac{\pi^{n / 2}}{\Gamma(1+n / 2)} \quad \text { and } \quad \omega_{n}:=n \kappa_{n}=\frac{2 \pi^{n / 2}}{\Gamma(n / 2)} .
$$

As usual, $\Gamma$ denotes the gamma function.

Date: 28 October 2018. Revised: 19 December 2018, 15 January 2019, 24 January 2019, and 19 March 2019.

2010 Mathematics Subject Classification. Primary: 52A39; 52A20. Secondary: 94A17; 52A22.

Key words and phrases. Alexandrov-Fenchel inequality, concentration, convex body, entropy, information theory, intrinsic volume, log-concave distribution, quermassintegral, ultra-log-concave sequence. 
1.2. The Intrinsic Volumes. In this section, we introduce the intrinsic volumes, their properties, and connections to other geometric functionals. A good reference for this material is [Sch14]. Intrinsic volumes are basic tools in stochastic and integral geometry [SWo8], and they appear in the study of random fields [ATo7].

We begin with a geometrically intuitive definition.

Definition 1.1 (Intrinsic Volumes). For each index $j=0,1,2, \ldots, n$, let $\boldsymbol{P}_{j} \in \mathbb{R}^{n \times n}$ be the orthogonal projector onto a fixed $j$-dimensional subspace of $\mathbb{R}^{n}$. Draw a rotation matrix $Q \in \mathbb{R}^{n \times n}$ uniformly at random (from the Haar measure on the compact, homogeneous group of $n \times n$ orthogonal matrices with determinant one). The intrinsic volumes of the nonempty convex body $\mathrm{K} \subset \mathbb{R}^{n}$ are the quantities

$$
V_{j}(\mathrm{~K}):=\left(\begin{array}{c}
n \\
j
\end{array}\right) \frac{\kappa_{n}}{\kappa_{j} \kappa_{n-j}} \mathbb{E}_{\boldsymbol{Q}}\left[\operatorname{Vol}_{j}\left(\boldsymbol{P}_{j} \boldsymbol{Q K}\right)\right] .
$$

We write $\mathbb{E}$ for expectation and $\mathbb{E}_{X}$ for expectation with respect to a specific random variable $X$. The intrinsic volumes of the empty set are identically zero: $V_{j}(\emptyset)=0$ for each index $j$.

Up to scaling, the $j$ th intrinsic volume is the average volume of a projection of the convex body onto a $j$-dimensional subspace, chosen uniformly at random. Following Federer [Fed59], we have chosen the normalization in (1.2) to remove the dependence on the dimension in which the convex body is embedded. McMullen [McM75] introduced the term "intrinsic volumes". In her work, Chevet [Che76] called $V_{j}$ the $j$-ième épaisseur or the " $j$ th thickness".

Example 1.2 (The Euclidean Ball). We can easily calculate the intrinsic volumes of the Euclidean unit ball because each projection is simply a Euclidean unit ball of lower dimension. Thus,

$$
V_{j}\left(\mathrm{~B}_{n}\right)=\left(\begin{array}{l}
n \\
j
\end{array}\right) \frac{\kappa_{n}}{\kappa_{n-j}} \text { for } j=0,1,2, \ldots, n .
$$

Example 1.3 (The Cube). We can also determine the intrinsic volumes of a cube:

$$
V_{j}\left(\mathrm{Q}_{n}\right)=\left(\begin{array}{c}
n \\
j
\end{array}\right) \text { for } j=0,1,2, \ldots, n .
$$

See Section 5 for the details of the calculation. A classic reference is [Sano4, pp. 224-227].

1.2.1. Geometric Functionals. The intrinsic volumes are closely related to familiar geometric functionals. The intrinsic volume $V_{0}$ is called the Euler characteristic; it takes the value zero for the empty set and the value one for each nonempty convex body. The intrinsic volume $V_{1}$ is proportional to the mean width, scaled so that $V_{1}\left([0,1] \times\left\{\mathbf{0}_{n-1}\right\}\right)=1$. Meanwhile, $V_{n-1}$ is half the surface area, and $V_{n}$ coincides with the ordinary volume measure, $\mathrm{Vol}_{n}$.

1.2.2. Properties. The intrinsic volumes satisfy many important properties. Let $\mathrm{C}, \mathrm{K} \subset \mathbb{R}^{n}$ be nonempty convex bodies. For each index $j=0,1,2, \ldots, n$, the intrinsic volume $V_{j}$ is...

(1) Nonnegative: $V_{j}(\mathrm{~K}) \geq 0$.

(2) Monotone: $C \subset \mathrm{K}$ implies $V_{j}(\mathrm{C}) \leq V_{j}(\mathrm{~K})$.

(3) Homogeneous: $V_{j}(\lambda \mathrm{K})=\lambda^{j} V_{j}(\mathrm{~K})$ for each $\lambda \geq 0$.

(4) Invariant: $V_{j}(\boldsymbol{T} \mathrm{K})=V_{j}(\mathrm{~K})$ for each proper rigid motion $\boldsymbol{T}$. That is, $\boldsymbol{T}$ acts by rotation and translation.

(5) Intrinsic: $V_{j}(\mathrm{~K})=V_{j}\left(\mathrm{~K} \times\left\{\mathbf{0}_{m}\right\}\right)$ for each natural number $m$.

(6) A Valuation: $V_{j}(\emptyset)=0$. If $\mathrm{C} \cup \mathrm{K}$ is also a convex body, then

$$
V_{j}(\mathrm{C} \cap \mathrm{K})+V_{j}(\mathrm{C} \cup \mathrm{K})=V_{j}(\mathrm{C})+V_{j}(\mathrm{~K}) \text {. }
$$

(7) Continuous: If $\mathrm{K}_{m} \rightarrow \mathrm{K}$ in the Hausdorff metric, then $V_{j}\left(\mathrm{~K}_{m}\right) \rightarrow V_{j}(\mathrm{~K})$.

With sufficient energy, one may derive all of these facts directly from Definition 1.1. See the books [KR97, San04, Gruo7, SWo8, Sch14] for further information about intrinsic volumes and related matters. 
1.2.3. Hadwiger's Characterization Theorems. Hadwiger [Had51, Had52, Had57] proved several wonderful theorems that characterize the intrinsic volumes. To state these results, we need a short definition. A valuation $F$ on $\mathbb{R}^{n}$ is simple if $F(\mathrm{~K})=0$ whenever $\operatorname{dim} \mathrm{K}<n$.

Fact 1.4 (Uniqueness of Volume). Suppose that $F$ is a simple, invariant, continuous valuation on convex bodies in $\mathbb{R}^{n}$. Then $F$ is a scalar multiple of the intrinsic volume $V_{n}$.

Fact 1.5 (The Basis of Intrinsic Volumes). Suppose that $F$ is an invariant, continuous valuation on convex bodies in $\mathbb{R}^{n}$. Then $F$ is a linear combination of the intrinsic volumes $V_{0}, V_{1}, V_{2}, \ldots, V_{n}$.

Together, these theorems demonstrate the fundamental importance of intrinsic volumes in convex geometry. They also construct a bridge to the field of integral geometry, which provides explicit formulas for geometric functionals defined by integrating over geometric groups (e.g., the family of proper rigid motions).

1.2.4. Quermassintegrals. With a different normalization, the mean projection volume appearing in (1.2) is also known as a quermassintegral. The relationship between the quermassintegrals and the intrinsic volumes is

$$
\left(\begin{array}{c}
n \\
j
\end{array}\right) W_{j}^{(n)}(\mathrm{K}):=\kappa_{j} V_{n-j}(\mathrm{~K}) \text { for } j=0,1,2, \ldots, n .
$$

The notation reflects the fact that the quermassintegral $W_{j}^{(n)}$ depends on the ambient dimension $n$, while the intrinsic volume does not.

1.3. The Intrinsic Volume Random Variable. In view of Example 1.3, we see that the intrinsic volume sequence of the cube $\mathrm{Q}_{n}$ is sharply peaked (around index $n / 2$ ). Example 1.2 shows that intrinsic volumes of the Euclidean ball $\mathrm{B}_{n}$ drop off quickly (starting around index $\sqrt{2 \pi n}$ ). This observation motivates us to ask whether the intrinsic volumes of a general convex body also exhibit some type of concentration.

It is natural to apply probabilistic methods to address this question. To that end, we first need to normalize the intrinsic volumes to construct a probability distribution.

Definition 1.6 (Normalized Intrinsic Volumes). The total intrinsic volume of the convex body K, also known as the Wills functional [Wil73, Had75, McM75], is the quantity

$$
W(\mathrm{~K}):=\sum_{j=0}^{n} V_{j}(\mathrm{~K}) .
$$

The normalized intrinsic volumes compose the sequence

$$
\tilde{V}_{j}(\mathrm{~K}):=\frac{V_{j}(\mathrm{~K})}{W(\mathrm{~K})} \quad \text { for } j=0,1,2, \ldots, n .
$$

In particular, the sequence $\left\{\tilde{V}_{j}(\mathrm{~K}): j=0,1,2, \ldots, n\right\}$ forms a probability distribution.

In spite of the similarity of notation, the total intrinsic volume $W$ should not be confused with a quermassintegral.

We may now construct a random variable that reflects the distribution of the intrinsic volumes of a convex body.

Definition 1.7 (Intrinsic Volume Random Variable). The intrinsic volume random variable $Z_{\mathrm{K}}$ associated with a convex body $\mathrm{K}$ takes nonnegative integer values according to the distribution

$$
\mathbb{P}\left\{Z_{\mathrm{K}}=j\right\}=\tilde{V}_{j}(\mathrm{~K}) \text { for } j=0,1,2, \ldots, n .
$$

The mean of the intrinsic volume random variable plays a special role in the analysis, so we exalt it with its own name and notation. 
Definition 1.8 (Central Intrinsic Volume). The central intrinsic volume of the convex body $\mathrm{K}$ is the quantity

$$
\Delta(\mathrm{K}):=\mathbb{E} Z_{\mathrm{K}}=\sum_{j=0}^{n} j \cdot \tilde{V}_{j}(\mathrm{~K})
$$

Equivalently, the central intrinsic volume is the centroid of the sequence of intrinsic volumes.

Since the intrinsic volume sequence of a convex body $\mathrm{K} \subset \mathbb{R}^{n}$ is supported on $\{0,1,2, \ldots, n\}$, it is immediate that the central intrinsic volume satisfies $\Delta(\mathrm{K}) \in[0, n]$. The extreme $n$ is unattainable (because a nonempty convex body has Euler characteristic $V_{0}(\mathrm{~K})=1$ ). But it is easy to construct examples that achieve values across the rest of the range.

Example 1.9 (The Scaled Cube). Fix $s \in[0, \infty)$. Using Example 1.3 and the homogeneity of intrinsic volumes, we see that total intrinsic volume of the scaled cube is

$$
W\left(s \mathrm{Q}_{n}\right)=\sum_{j=0}^{n}\left(\begin{array}{l}
n \\
j
\end{array}\right) \cdot s^{j}=(1+s)^{n} .
$$

The central intrinsic volume of the scaled cube is

$$
\Delta\left(s \mathrm{Q}_{n}\right)=\frac{1}{(1+s)^{n}} \sum_{j=0}^{n} j \cdot\left(\begin{array}{c}
n \\
j
\end{array}\right) \cdot s^{j}=\sum_{j=0}^{n} j \cdot\left(\begin{array}{c}
n \\
j
\end{array}\right) \cdot\left(\frac{s}{1+s}\right)^{j}\left(1-\frac{s}{1+s}\right)^{n-j}=\frac{n s}{1+s} .
$$

We recognize the mean of the random variable $\operatorname{BIN}(s /(1+s), n)$ to reach the last identity. Note that the quantity $\Delta\left(s \mathrm{Q}_{n}\right)=n s /(1+s)$ sweeps through the interval $[0, n)$ as we vary $s \in[0, \infty)$.

Example 1.10 (Large Sets). More generally, we can compute the limits of the normalized intrinsic volumes of a growing set:

$$
\begin{array}{ll}
\lim _{s \rightarrow \infty} \tilde{V}_{j}(s \mathrm{~K}) \rightarrow 0 & \text { for } j<\operatorname{dim} \mathrm{K} \\
\lim _{s \rightarrow \infty} \tilde{V}_{j}(s \mathrm{~K}) \rightarrow 1 & \text { for } j=\operatorname{dim} \mathrm{K} .
\end{array}
$$

This point follows from the homogeneity of intrinsic volumes, noted in Section 1.2.2.

1.4. Concentration of Intrinsic Volumes. Our main result states that the intrinsic volume random variable concentrates sharply around the central intrinsic volume.

Theorem 1.11 (Concentration of Intrinsic Volumes). Let $\mathrm{K} \subset \mathbb{R}^{n}$ be a nonempty convex body with intrinsic volume random variable $Z_{\mathrm{K}}$. The variance satisfies

$$
\operatorname{Var}\left[Z_{\mathrm{K}}\right] \leq 4 n \text {. }
$$

Furthermore, in the range $0 \leq t \leq \sqrt{n}$, we have the tail inequality

$$
\mathbb{P}\left\{\left|Z_{\mathrm{K}}-\mathbb{E} Z_{\mathrm{K}}\right| \geq t \sqrt{n}\right\} \leq 2 \mathrm{e}^{-3 t^{2} / 28} .
$$

To prove this theorem, we first convert questions about the intrinsic volume random variable into questions about metric geometry (Section 2). We reinterpret the metric geometry formulations in terms of the information content of a log-concave probability density. Then we can control the variance (Section 3) and concentration properties (Section 4) of the intrinsic volume random variable using the analogous results for the information content random variable.

A general probability distribution on $\{0,1,2, \ldots, n\}$ can have variance higher than $n^{2} / 3$. In contrast, the intrinsic volume random variable has variance no greater than $4 n$. Moreover, the intrinsic volume random variable behaves, at worst, like a normal random variable with mean $\mathbb{E} Z_{\mathrm{K}}$ and variance less than $5 \mathrm{n}$. Thus, most of the mass of the intrinsic volume sequence is concentrated on an interval of about $O(\sqrt{n})$ indices.

Looking back to Example 1.3, concerning the unit-volume cube $Q_{n}$, we see that Theorem 1.11 gives a qualitatively accurate description of the intrinsic volume sequence. On the other hand, the bounds for scaled cubes $s \mathrm{Q}_{n}$ can be quite poor; see Section 5.3. 
1.5. Concentration of Conic Intrinsic Volumes. Theorem 1.11 and its proof parallel recent developments in the theory of conic intrinsic volumes, which appear in the papers [ALMT14, MT14a, GNP17]. Using the concentration of conic intrinsic volumes, we were able to establish that random configurations of convex cones exhibit striking phase transitions; these facts have applications in signal processing [McC13, MT14b, ALMT14, MT17]. We are confident that extending the ideas in the current paper will help us discover new phase transition phenomena in Euclidean integral geometry.

1.6. Maximum-Entropy Convex Bodies. The probabilistic approach to the intrinsic volume sequence suggests other questions to investigate. For instance, we can study the entropy of the intrinsic volume random variable, which reflects the dispersion of the intrinsic volume sequence.

Definition 1.12 (Intrinsic Entropy). Let $K \subset \mathbb{R}^{n}$ be a nonempty convex body. The intrinsic entropy of $\mathrm{K}$ is the entropy of the intrinsic volume random variable $Z_{\mathrm{K}}$ :

$$
\operatorname{IntEnt}(\mathrm{K}):=\operatorname{Ent}\left[Z_{\mathrm{K}}\right]=-\sum_{j=0}^{n} \tilde{V}_{j}(\mathrm{~K}) \cdot \log \tilde{V}_{j}(\mathrm{~K}) .
$$

We have the following extremal result.

Theorem 1.13 (Cubes Have Maximum Entropy). Fix the ambient space $\mathbb{R}^{n}$, and let $d \in[0, n)$. There is a scaled cube whose central intrinsic volume equals $d$ :

$$
\Delta\left(s_{d, n} \mathrm{Q}_{n}\right)=d \quad \text { when } \quad s_{d, n}=\frac{d}{n-d} .
$$

Among convex bodies with central intrinsic volume $d$, the scaled cube $s_{d, n} \mathrm{Q}_{n}$ has the maximum intrinsic entropy. Among all convex bodies, the unit-volume cube has the maximum intrinsic entropy. In symbols,

$$
\max \{\operatorname{IntEnt}(\mathrm{K}): \Delta(\mathrm{K})=d\}=\operatorname{IntEnt}\left(s_{d, n} \mathrm{Q}_{n}\right) \leq \operatorname{IntEnt}\left(\mathrm{Q}_{n}\right) .
$$

The maximum takes place over all nonempty convex bodies $\mathrm{K} \subset \mathbb{R}^{n}$.

The proof of Theorem 1.13 also depends on recent results from information theory, as well as some deep properties of the intrinsic volume sequence. This analysis appears in Section 6.

Theorem 1.13 joins a long procession of results on the extremal properties of the cube. In particular, the cube solves the (affine) reverse isoperimetric problem for symmetric convex bodies [Bal91]. That is, every symmetric convex body $\mathrm{K} \subset \mathbb{R}^{n}$ has an affine image whose volume is one and whose surface area is not greater than $2 n$, the surface area of $Q_{n}$. See Section 1.7.2 for an equivalent statement.

Remark 1.14 (Minimum Entropy). The convex body consisting of a single point $\boldsymbol{x}_{0} \in \mathbb{R}^{n}$ has the minimum intrinsic entropy: $\operatorname{IntEnt}\left(\left\{\boldsymbol{x}_{0}\right\}\right)=0$. Very large convex bodies also have negligible entropy:

$$
\lim _{s \rightarrow \infty} \operatorname{IntEnt}(s \mathrm{~K})=0 \quad \text { for each nonempty convex body } \mathrm{K} \subset \mathbb{R}^{n} .
$$

The limit is a consequence of Example 1.10.

1.7. Other Inequalities for Intrinsic Volumes. The classic literature on convex geometry contains a number of prominent inequalities relating the intrinsic volumes, and this topic continues to arouse interest. This section offers a short overview of the main results of this type. Our presentation is influenced by [McM91, PPV17]. See [Sch14, Chap. 7] for a comprehensive treatment.

Remark 1.15 (Unrelated work). Although the title of the paper [AS16] includes the phrase "concentration of intrinsic volumes," the meaning is quite different. Indeed, the focus of that work is to study hyperplane arrangements via the intrinsic volumes of a random sequence associated with the arrangement. 
1.7.1. Ultra-Log-Concavity. The Alexandrov-Fenchel inequality (AFI) is a profound result on the behavior of mixed volumes; see [Sch14, Sec. 7.3] or [SH18]. We can specialize the AFI from mixed volumes to the particular case of quermassintegrals. In this instance, the AFI states that the quermassintegrals of a convex body $\mathrm{K} \subset \mathbb{R}^{n}$ compose a log-concave sequence:

$$
W_{j}^{(n)}(\mathrm{K})^{2} \geq W_{j+1}^{(n)}(\mathrm{K}) \cdot W_{j-1}^{(n)}(\mathrm{K}) \quad \text { for } j=1,2,3, \ldots, n-1 .
$$

As Chevet [Che76] and McMullen [McM91] independently observed, the log-concavity (1.6) of the quermassintegral sequence implies that the intrinsic volumes form an ultra-log-concave (ULC) sequence:

$$
j \cdot V_{j}(\mathrm{~K})^{2} \geq(j+1) \cdot V_{j+1}(\mathrm{~K}) \cdot V_{j-1}(\mathrm{~K}) \text { for } j=1,2,3, \ldots, n-1 .
$$

This fact plays a key role in the proof of Theorem 1.13. For more information on log-concavity and ultra-log-concavity, see the survey article [SW14].

From (1.7), Chevet and McMullen both deduce that all of the intrinsic volumes are controlled by the first one, and they derive an estimate for the total intrinsic volume:

$$
V_{j}(\mathrm{~K}) \leq \frac{1}{j !} V_{1}(\mathrm{~K})^{j} \quad \text { for } j=1,2,3, \ldots, n \text {, hence } W(\mathrm{~K}) \leq \mathrm{e}^{V_{1}(\mathrm{~K})} .
$$

This estimate implies some growth and decay properties of the intrinsic volume sequence. An interesting application appears in Vitale's paper [Vit96], which derives concentration for the supremum of a Gaussian process from the foregoing bound on the total intrinsic volume.

It is possible to establish a concentration result for intrinsic volumes as a direct consequence of (1.7). Indeed, it is intuitive that a ULC sequence should concentrate around its centroid. This point follows from Caputo et al. [CDPPo9, Sec. 3.2], which transcribes the usual semigroup proof of a log-Sobolev inequality to the discrete setting. When applied to intrinsic volumes, this method gives concentration on the scale of the mean width $V_{1}(\mathrm{~K})$ of the convex body $\mathrm{K}$. This result captures a phenomenon different from Theorem 1.11, where the scale for the concentration is the dimension $n$.

1.7.2. Isoperimetric Ratios. Another classical consequence of the AFI is a sequence of comparisons for the isoperimetric ratios of the volume of a convex body $\mathrm{K} \subset \mathbb{R}^{n}$, relative to the Euclidean ball $\mathrm{B}_{n}$ :

$$
\left(\frac{V_{n}(\mathrm{~K})}{V_{n}\left(\mathrm{~B}_{n}\right)}\right)^{1 / n} \leq\left(\frac{V_{n-1}(\mathrm{~K})}{V_{n-1}\left(\mathrm{~B}_{n}\right)}\right)^{1 /(n-1)} \leq \cdots \leq \frac{V_{1}(\mathrm{~K})}{V_{1}\left(\mathrm{~B}_{n}\right)}
$$

The first inequality is the isoperimetric inequality, and the inequality between $V_{n}$ and $V_{1}$ is called Urysohn's inequality [Sch14, Sec. 7.2]. Isoperimetric ratios play a prominent role in asymptotic convex geometry; for example, see [Pis89, Bal97, AAGM15].

Some of the inequalities in (1.8) can be inverted by applying affine transformations. For example, Ball's reverse isoperimetric inequality [Bal91] states that $\mathrm{K}$ admits an affine image $\hat{\mathrm{K}}$ for which

$$
\left(\frac{V_{n-1}(\hat{\mathrm{K}})}{V_{n-1}\left(\mathrm{~B}_{n}\right)}\right)^{1 /(n-1)} \leq \text { const }_{n} \cdot\left(\frac{V_{n}(\hat{\mathrm{K}})}{V_{n}\left(\mathrm{~B}_{n}\right)}\right)^{1 / n}
$$

The sharp value for the constant is known; equality holds when $\mathrm{K}$ is a simplex. If we restrict our attention to symmetric convex bodies, then the cube is extremal.

The recent paper [PPV17] of Paouris et al. contains a more complete, but less precise, set of reversals. Suppose that $\mathrm{K}$ is a symmetric convex body. Then there is a parameter $\beta_{\star}:=\beta_{\star}(\mathrm{K})$ for which

$$
\frac{V_{1}(\mathrm{~K})}{V_{1}\left(\mathrm{~B}_{n}\right)} \leq\left[1+\mathrm{const} \cdot\left(\beta_{\star} j \log \left(\frac{\mathrm{e}}{j \beta_{\star}}\right)\right)^{1 / 2}\right] \cdot\left(\frac{V_{j}(\mathrm{~K})}{V_{j}\left(\mathrm{~B}_{n}\right)}\right)^{1 / j} \quad \text { for } j=1,2,3, \ldots, \text { const } / \beta_{\star} \text {. }
$$

The constants here are universal but unspecified. This result implies that the prefix of the sequence of isoperimetric ratios is roughly constant. The result (1.9) leaves open the question about the behavior of the sequence beyond the distinguished point. 
It would be interesting to reconcile the work of Paouris et al. [PPV17] with Theorem 1.11. In particular, it is unclear whether the isoperimetric ratios remain constant, or whether they exhibit some type of phase transition. We believe that our techniques have implications for this question.

\section{STEINER's Formula AND Distance InTEgRALS}

The first step in our program is to convert questions about the intrinsic volume random variable into questions in metric geometry. We can accomplish this goal using Steiner's formula, which links the intrinsic volumes of a convex body to its expansion properties. We reinterpret Steiner's formula as a distance integral, and we use this result to compute moments of the intrinsic volume random variable. This technique, which appears to be novel, drives our approach.

2.1. Steiner's Formula. The Minkowski sum of a nonempty convex body and a Euclidean ball is called a parallel body. Steiner's formula gives an explicit expansion for the volume of the parallel body in terms of the intrinsic volumes of the convex body.

Fact 2.1 (Steiner's Formula). Let $\mathrm{K} \subset \mathbb{R}^{n}$ be a nonempty convex body. For each $\lambda \geq 0$,

$$
\operatorname{Vol}_{n}\left(\mathrm{~K}+\lambda \mathrm{B}_{n}\right)=\sum_{j=0}^{n} \lambda^{n-j} \kappa_{n-j} V_{j}(\mathrm{~K})
$$

In other words, the volume of the parallel body is a polynomial function of the expansion radius. Moreover, the coefficients depend only on the intrinsic volumes of the convex body. The proof of Fact 2.1 is fairly easy; see [Sch14, Gruo7].

Remark 2.2 (Steiner and Kubota). Steiner's formula can be used to define the intrinsic volumes. The definition we have given in (1.2) is usually called Kubota's formula; it can be derived as a consequence of Fact 2.1 and Cauchy's formula for surface area. For example, see [AAGM15, Sec. B.5].

2.2. Distance Integrals. The parallel body can also be expressed as the set of points within a fixed distance of the convex body. This observation motivates us to introduce the distance to a convex set.

Definition 2.3 (Distance to a Convex Body). The distance to a nonempty convex body $\mathrm{K}$ is the function

$$
\operatorname{dist}(\boldsymbol{x}, \mathrm{K}):=\min \{\|\boldsymbol{y}-\boldsymbol{x}\|: \boldsymbol{y} \in \mathrm{K}\} \quad \text { where } \boldsymbol{x} \in \mathbb{R}^{n} \text {. }
$$

It is not hard to show that the distance, $\operatorname{dist}(\cdot, \mathrm{K})$, and its square, $\operatorname{dist}^{2}(\cdot, \mathrm{K})$, are both convex functions.

Here is an alternative statement of Steiner's formula in terms of distance integrals [Had75].

Proposition 2.4 (Distance Integrals). Let $\mathrm{K} \subset \mathbb{R}^{n}$ be a nonempty convex body. Let $f: \mathbb{R}_{+} \rightarrow \mathbb{R}$ be an absolutely integrable function. Provided that the integrals on the right-hand side converge,

$$
\int_{\mathbb{R}^{n}} f(\operatorname{dist}(\boldsymbol{x}, \mathrm{K})) \mathrm{d} \boldsymbol{x}=f(0) \cdot V_{n}(\mathrm{~K})+\sum_{j=0}^{n-1}\left(\omega_{n-j} \int_{0}^{\infty} f(r) \cdot r^{n-j-1} \mathrm{~d} r\right) \cdot V_{j}(\mathrm{~K}) .
$$

This result is equivalent to Fact 2.1.

Proof. For $r>0$, Steiner's formula gives an expression for the volume of the locus of points within distance $r$ of the convex body:

$$
\operatorname{Vol}_{n}\left\{\boldsymbol{x} \in \mathbb{R}^{n}: \operatorname{dist}(\boldsymbol{x}, \mathrm{K}) \leq r\right\}=\sum_{j=0}^{n} r^{n-j} \kappa_{n-j} V_{j}(\mathrm{~K}) .
$$

The rate of change in this volume satisfies

$$
\frac{\mathrm{d}}{\mathrm{d} r} \operatorname{Vol}_{n}\left\{\boldsymbol{x} \in \mathbb{R}^{n}: \operatorname{dist}(\boldsymbol{x}, \mathrm{K}) \leq r\right\}=\sum_{j=0}^{n-1} r^{n-j-1} \omega_{n-j} V_{j}(\mathrm{~K}) .
$$

We have used the relation (1.1) that $\omega_{n-j}=(n-j) \kappa_{n-j}$. is,

Let $\mu_{\sharp}$ be the push-forward of the Lebesgue measure on $\mathbb{R}^{n}$ to $\mathbb{R}_{+}$by the function $\operatorname{dist}(\cdot ; \mathrm{K})$. That

$$
\mu_{\sharp}(\mathrm{A}):=\operatorname{Vol}_{n}\left\{\boldsymbol{x} \in \mathbb{R}^{n}: \operatorname{dist}(\boldsymbol{x} ; \mathrm{K}) \in \mathrm{A}\right\} \quad \text { for each Borel set } \mathrm{A} \subset \mathbb{R}_{+} \text {. }
$$


This measure clearly satisfies $\mu_{\sharp}(\{0\})=V_{n}(\mathrm{~K})$. Beyond that, when $0<a<b$,

$$
\begin{aligned}
\mu_{\sharp}((a, b]) & =\operatorname{Vol}_{n}\left\{\boldsymbol{x} \in \mathbb{R}^{n}: a<\operatorname{dist}(\boldsymbol{x} ; \mathrm{K}) \leq b\right\} \\
& =\operatorname{Vol}_{n}\left\{\boldsymbol{x} \in \mathbb{R}^{n}: \operatorname{dist}(\boldsymbol{x} ; \mathrm{K}) \leq b\right\}-\operatorname{Vol}_{n}\left\{\boldsymbol{x} \in \mathbb{R}^{n}: \operatorname{dist}(\boldsymbol{x} ; \mathrm{K}) \leq a\right\} \\
& =\int_{a}^{b} \frac{\mathrm{d}}{\mathrm{d} r} \operatorname{Vol}_{n}\left\{\boldsymbol{x} \in \mathbb{R}^{n}: \operatorname{dist}(\boldsymbol{x} ; \mathrm{K}) \leq r\right\} \mathrm{d} r .
\end{aligned}
$$

Therefore, by definition of the push-forward,

$$
\begin{aligned}
\int_{\mathbb{R}^{n}} f(\operatorname{dist}(\boldsymbol{x} ; \mathrm{K})) \mathrm{d} \boldsymbol{x} & =\int_{\mathbb{R}_{+}} f(r) \mathrm{d} \mu_{\sharp}(r) \\
& =f(0) \cdot V_{n}(\mathrm{~K})+\int_{0}^{\infty} f(r) \cdot \frac{\mathrm{d}}{\mathrm{d} r} \operatorname{Vol}_{n}\left\{\boldsymbol{x} \in \mathbb{R}^{n}: \operatorname{dist}(\boldsymbol{x} ; \mathrm{K}) \leq r\right\} \mathrm{d} r .
\end{aligned}
$$

Introduce (2.1) into the last display to arrive at the result.

2.3. Moments of the Intrinsic Volume Sequence. We can compute moments (i.e., linear functionals) of the sequence of intrinsic volumes by varying the function $f$ in Proposition 2.4. To that end, it is helpful to make another change of variables.

Corollary 2.5 (Distance Integrals II). Let $\mathrm{K} \subset \mathbb{R}^{n}$ be a nonempty convex body. Let $g: \mathbb{R}_{+} \rightarrow \mathbb{R}$ be an absolutely integrable function. Provided the integrals on the right-hand side converge,

$$
\begin{aligned}
\int_{\mathbb{R}^{n}} g\left(\pi \operatorname{dist}^{2}(\boldsymbol{x}, \mathrm{K})\right) & \cdot \mathrm{e}^{-\pi \operatorname{dist}^{2}(\boldsymbol{x}, \mathrm{K})} \mathrm{d} \boldsymbol{x} \\
& =g(0) \cdot V_{n}(\mathrm{~K})+\sum_{j=0}^{n-1}\left(\frac{1}{\Gamma((n-j) / 2)} \int_{0}^{\infty} g(r) \cdot r^{-1+(n-j) / 2} \mathrm{e}^{-r} \mathrm{~d} r\right) \cdot V_{j}(\mathrm{~K}) .
\end{aligned}
$$

Proof. Set $f(r)=g\left(\pi r^{2}\right) \cdot \mathrm{e}^{-\pi r^{2}}$ in Proposition 2.4 and invoke (1.1).

We are now prepared to compute some specific moments of the intrinsic volume sequence by making special choices of $g$ in Corollary 2.5.

Example 2.6 (Total Intrinsic Volume). Consider the case where $g(r)=1$. We obtain the appealing formula

$$
\int_{\mathbb{R}^{n}} \mathrm{e}^{-\pi \operatorname{dist}^{2}(\boldsymbol{x}, \mathrm{K})} \mathrm{d} \boldsymbol{x}=\sum_{j=0}^{n} V_{j}(\mathrm{~K})=W(\mathrm{~K}) .
$$

The total intrinsic volume $W(\mathrm{~K})$ was defined in (1.3). This identity appears in [Had75, McM75].

Example 2.7 (Central Intrinsic Volume). The choice $g(r)=2 r / W(\mathrm{~K})$ yields

$$
\frac{1}{W(\mathrm{~K})} \int_{\mathbb{R}^{n}} 2 \pi \operatorname{dist}^{2}(\boldsymbol{x}, \mathrm{K}) \cdot \mathrm{e}^{-\pi \operatorname{dist}^{2}(\boldsymbol{x}, \mathrm{K})} \mathrm{d} \boldsymbol{x}=\frac{1}{W(\mathrm{~K})} \sum_{j=0}^{n}(n-j) \cdot V_{j}(\mathrm{~K})=n-\mathbb{E} Z_{\mathrm{K}} .
$$

We have recognized the total intrinsic volume (1.3) and the central intrinsic volume (1.5).

Example 2.8 (Generating Functions). We can also develop an expression for the generating function of the intrinsic volume sequence by selecting $g(r)=\mathrm{e}^{\left(1-\lambda^{2}\right) r}$. Thus,

$$
\int_{\mathbb{R}^{n}} \mathrm{e}^{-\lambda^{2} \pi \operatorname{dist}^{2}(\boldsymbol{x}, \mathrm{K})} \mathrm{d} \boldsymbol{x}=\lambda^{-n} \sum_{j=0}^{n} \lambda^{j} V_{j}(\mathrm{~K}) .
$$

This expression is valid for all $\lambda>0$. See [Had75] or [SWo8, Lem. 14.2.1].

We can reframe the relation (2.2) in terms of the moment generating function of the intrinsic volume random variable $Z_{\mathrm{K}}$. To do so, we make the change of variables $\lambda=\mathrm{e}^{\theta}$ and divide by the total intrinsic volume $W(\mathrm{~K})$ :

$$
\mathbb{E} \mathrm{e}^{\theta\left(Z_{\mathrm{K}}-n\right)}=\frac{1}{W(\mathrm{~K})} \int_{\mathbb{R}^{n}} \mathrm{e}^{-\mathrm{e}^{2 \theta} \pi \operatorname{dist}^{2}(\boldsymbol{x}, \mathrm{K})} \mathrm{d} \boldsymbol{x} .
$$


This expression remains valid for all $\theta \in \mathbb{R}$.

Remark 2.9 (Other Moments). In fact, we can compute any moment of the intrinsic volume sequence by selecting an appropriate function $f$ in Proposition 2.4. Corollary 2.5 is designed to produce gamma integrals. Beta integrals also arise naturally and lead to other striking relations. For instance,

$$
\int_{\mathbb{R}^{n}} \frac{\mathrm{d} \boldsymbol{x}}{(1+\lambda \operatorname{dist}(\boldsymbol{x}, \mathrm{K}))^{n+1}}=\kappa_{n} \lambda^{-n} \sum_{j=0}^{n} \lambda^{j} \frac{V_{j}(\mathrm{~K})}{V_{j}\left(\mathrm{~B}_{n}\right)} \text { for } \lambda>0 .
$$

The intrinsic volumes of the Euclidean ball are computed in Example 1.2. Isoperimetric ratios appear naturally in convex geometry (see Section 1.7.2), so this type of result may have independent interest.

\section{VARiance of the Intrinsic Volume Random Variable}

Let us embark on our study of the intrinsic volume random variable. The main result of this section states that the variance of the intrinsic volume random variable is significantly smaller than its range. This is a more precise version of the variance bound in Theorem 1.11.

Theorem 3.1 (Variance of the Intrinsic Volume Random Variable). Let $\mathrm{K} \subset \mathbb{R}^{n}$ be a nonempty convex body with intrinsic volume random variable $Z_{\mathrm{K}}$. We have the inequalities

$$
\operatorname{Var}\left[Z_{\mathrm{K}}\right] \leq 2\left(n+\mathbb{E} Z_{\mathrm{K}}\right) \leq 4 n .
$$

The proof of Theorem 3.1 occupies the rest of this section. We make a connection between the distance integrals from Section 2 and the information content of a log-concave probability measure. By using recent results on the variance of information, we can develop bounds for the distance integrals. These results, in turn, yield bounds on the variance of the intrinsic volume random variable. A closely related argument, appearing in Section 4, produces exponential concentration.

Remark 3.2 (An Alternative Argument). Theorem 3.1 can be sharpened using variance inequalities for log-concave densities. Indeed, it holds that

$$
\operatorname{Var}\left[Z_{\mathrm{K}}\right] \leq 2\left(n-\mathbb{E} Z_{\mathrm{K}}\right) \text {. }
$$

To prove this claim, we apply the Brascamp-Lieb inequality [BL76, Thm. 4.1] to a perturbation of the log-concave density (3.4) described below. It is not clear whether similar ideas lead to normal concentration (because the density is not strongly log-concave), so we have chosen to omit this development.

3.1. The Varentropy of a Log-Concave Distribution. First, we outline some facts from information theory about the information content in a log-concave random variable. Let $\mu: \mathbb{R}^{n} \rightarrow \mathbb{R}_{+}$be a log-concave probability density; that is, a probability density that satisfies the inequalities

$$
\mu(\tau \boldsymbol{x}+(1-\tau) \boldsymbol{y}) \geq \mu(\boldsymbol{x})^{\tau} \mu(\boldsymbol{y})^{1-\tau} \text { for } \boldsymbol{x}, \boldsymbol{y} \in \mathbb{R}^{n} \text { and } \tau \in[0,1] .
$$

We define the information content $I_{\mu}$ of a random point drawn from the density $\mu$ to be the random variable

$$
I_{\mu}:=-\log \mu(\boldsymbol{y}) \quad \text { where } \quad \boldsymbol{y} \sim \mu .
$$

The symbol $\sim$ means "has the distribution." The terminology is motivated by the operational interpretation of the information content of a discrete random variable as the number of bits required to represent a random realization using a code with minimal average length [BM11].

The expected information content $\mathbb{E} I_{\mu}$ is usually known as the entropy of the distribution $\mu$. The varentropy of the distribution is the variance of information content:

$$
\operatorname{VarEnt}[\mu]:=\operatorname{Var}\left[I_{\mu}\right]=\mathbb{E}\left(I_{\mu}-\mathbb{E} I_{\mu}\right)^{2} .
$$

Here and elsewhere, nonlinear functions bind before the expectation.

Bobkov \& Madiman [BM11] showed that the varentropy of a log-concave distribution on $\mathbb{R}^{n}$ is not greater than a constant multiple of $n$. Other researchers quickly determined the optimal constant. 
The following result was obtained independently by Nguyen [Ngu13] and by Wang [Wan14] in their doctoral dissertations.

Fact 3.3 (Varentropy of a Log-Concave Distribution). Let $\mu: \mathbb{R}^{n} \rightarrow \mathbb{R}_{+}$be a log-concave probability density. Then

$$
\operatorname{Var} \operatorname{Ent}[\mu] \leq n
$$

See Fradelizi et al. [FMW16] for more background and a discussion of this result.

For future reference, note that the varentropy and related quantities exhibit a simple scale invariance. Consider the shifted information content

$$
I_{c \mu}:=-\log (c \mu(\boldsymbol{y})) \quad \text { where } c>0 \text { and } \boldsymbol{y} \sim \mu .
$$

It follows from the definition that

$$
I_{c \mu}-\mathbb{E} I_{c \mu}=I_{\mu}-\mathbb{E} I_{\mu} \quad \text { for each } c>0 .
$$

In particular, $\operatorname{Var}\left[I_{c \mu}\right]=\operatorname{Var}\left[I_{\mu}\right]$.

3.2. A Log-Concave Density. Next, we observe that the central intrinsic volume is related to the information content of a log-concave density. For a nonempty convex body $\mathrm{K} \subset \mathbb{R}^{n}$, define

$$
\mu_{\mathrm{K}}(\boldsymbol{x}):=\frac{1}{W(\mathrm{~K})} \mathrm{e}^{-\pi \operatorname{dist}^{2}(\boldsymbol{x}, \mathrm{K})} \text { for } \boldsymbol{x} \in \mathbb{R}^{n} .
$$

The density $\mu_{\mathrm{K}}$ is log-concave because the squared distance to a convex body is a convex function. The calculation in Example 2.6 ensures that $\mu_{\mathrm{K}}$ is a probability density.

Introduce the (shifted) information content random variable associated with $\mathrm{K}$ :

$$
H_{\mathrm{K}}:=-\log \left(W(\mathrm{~K}) \cdot \mu_{\mathrm{K}}(\boldsymbol{y})\right)=\pi \operatorname{dist}^{2}(\boldsymbol{y}, \mathrm{K}) \quad \text { where } \quad \boldsymbol{y} \sim \mu_{\mathrm{K}} .
$$

Up to the presence of the factor $W(\mathrm{~K})$, the random variable $H_{\mathrm{K}}$ is the information content of a random draw from the distribution $\mu_{\mathrm{K}}$. In view of (3.2) and (3.3),

$$
\operatorname{Var}\left[H_{\mathrm{K}}\right]=\operatorname{Var}\left[I_{\mu_{\mathrm{K}}}\right]=\operatorname{VarEnt}\left[\mu_{\mathrm{K}}\right] .
$$

More generally, all central moments and cumulants of $H_{\mathrm{K}}$ coincide with the corresponding central moments and cumulants of $I_{\mu_{\mathrm{K}}}$ :

$$
\mathbb{E} f\left(H_{\mathrm{K}}-\mathbb{E} H_{\mathrm{K}}\right)=\mathbb{E} f\left(I_{\mu_{\mathrm{K}}}-\mathbb{E} I_{\mu_{\mathrm{K}}}\right) .
$$

This expression is valid for any function $f: \mathbb{R} \rightarrow \mathbb{R}$ such that the expectations exist.

3.3. Information Content and Intrinsic Volumes. We are now prepared to connect the moments of the intrinsic volume random variable $Z_{\mathrm{K}}$ with the moments of the information content random variable $H_{\mathrm{K}}$. These representations allow us to transfer results about information content into data about the intrinsic volumes.

Using the notation from the last section, Example 2.7 gives a relation between the expectations:

$$
\mathbb{E} Z_{\mathrm{K}}=n-2 \mathbb{E} H_{\mathrm{K}}
$$

The next result provides a similar relationship between the variances.

Proposition 3.4 (Variance of the Intrinsic Volume Random Variable). Let $\mathrm{K} \subset \mathbb{R}^{n}$ be a nonempty convex body with intrinsic volume random variable $Z_{\mathrm{K}}$ and information content random variable $H_{\mathrm{K}}$. We have the variance identity

$$
\operatorname{Var}\left[Z_{\mathrm{K}}\right]=4\left(\operatorname{Var}\left[H_{\mathrm{K}}\right]-\mathbb{E} H_{\mathrm{K}}\right)
$$


Proof. Apply Corollary 2.5 with the function $g(r)=4 r^{2} / W(\mathrm{~K})$ to obtain

$$
\begin{aligned}
4 \mathbb{E} H_{\mathrm{K}}^{2} & =\frac{1}{W(\mathrm{~K})} \int_{\mathbb{R}^{n}} 4 \pi^{2} \operatorname{dist}^{4}(\boldsymbol{x}, \mathrm{K}) \cdot \mathrm{e}^{-\pi \operatorname{dist}^{2}(\boldsymbol{x}, \mathrm{K})} \mathrm{d} \boldsymbol{x} \\
& =\frac{1}{W(\mathrm{~K})} \sum_{j=0}^{n-1}(n-j)((n-j)+2) \cdot V_{j}(\mathrm{~K}) \\
& =\mathbb{E}\left(n-Z_{\mathrm{K}}\right)^{2}+2 \mathbb{E}\left[n-Z_{\mathrm{K}}\right] \\
& =\operatorname{Var}\left[n-Z_{\mathrm{K}}\right]+\left(\mathbb{E}\left[n-Z_{\mathrm{K}}\right]\right)^{2}+2 \mathbb{E}\left[n-Z_{\mathrm{K}}\right] \\
& =\operatorname{Var}\left[Z_{\mathrm{K}}\right]+4\left(\mathbb{E} H_{\mathrm{K}}\right)^{2}+4 \mathbb{E} H_{\mathrm{K}} .
\end{aligned}
$$

We have used the definition (1.4) of the intrinsic volume random variable to express the sum as an expectation. In the last step, we used the relation (3.8) twice to pass to the random variable $H_{\mathrm{K}}$. Finally, rearrange the display to complete the proof.

3.4. Proof of Theorem 3.1. We may now establish the main result of this section. Proposition 3.4 yields

$$
\operatorname{Var}\left[Z_{\mathrm{K}}\right]=4\left(\operatorname{Var}\left[H_{\mathrm{K}}\right]-\mathbb{E} H_{\mathrm{K}}\right)=4 \operatorname{VarEnt}\left[\mu_{\mathrm{K}}\right]-2\left(n-\mathbb{E} Z_{\mathrm{K}}\right) \leq 2 n+2 \mathbb{E} Z_{\mathrm{K}} \leq 4 n .
$$

We have invoked (3.6) to replace the variance of $H_{\mathrm{K}}$ with the varentropy and (3.8) to replace $\mathbb{E} H_{\mathrm{K}}$ by the central intrinsic volume $\mathbb{E} Z_{\mathrm{K}}$. The inequality is a consequence of Fact 3.3, which controls the varentropy of the log-concave density $\mu_{\mathrm{K}}$. We obtain the final bound by noting that $\mathbb{E} Z_{\mathrm{K}} \leq n$.

Here is an alternative approach to the final bound that highlights the role of the varentropy:

$$
\operatorname{Var}\left[Z_{\mathrm{K}}\right] \leq 4 \operatorname{Var}\left[H_{\mathrm{K}}\right]=4 \operatorname{Var} \operatorname{Ent}\left[\mu_{\mathrm{K}}\right] \leq 4 n .
$$

The first inequality follows from Proposition 3.4, and the second inequality is Fact 3.3.

\section{Concentration of the Intrinsic Volume Random Variable}

The square root of the variance of the intrinsic volume random variable $Z_{\mathrm{K}}$ gives the scale for fluctuations about the mean. These fluctuations have size $O(\sqrt{n})$, which is much smaller than the $O(n)$ range of the random variable. This observation motivates us to investigate the concentration properties of $Z_{\mathrm{K}}$. In this section, we develop a refined version of the tail bound from Theorem 1.11.

Theorem 4.1 (Tail Bounds for Intrinsic Volumes). Let $\mathrm{K} \subset \mathbb{R}^{n}$ be a nonempty convex body with intrinsic volume random variable $Z_{\mathrm{K}}$. For all $t \geq 0$, we have the inequalities

$$
\begin{gathered}
\mathbb{P}\left\{Z_{\mathrm{K}}-\mathbb{E} Z_{\mathrm{K}} \geq t\right\} \leq \exp \left\{-\left(n+\mathbb{E} Z_{\mathrm{K}}\right) \cdot \psi^{*}\left(\frac{t}{n+\mathbb{E} Z_{\mathrm{K}}}\right)\right\} ; \\
\mathbb{P}\left\{Z_{\mathrm{K}}-\mathbb{E} Z_{\mathrm{K}} \leq-t\right\} \leq \exp \left\{-\left(n+\mathbb{E} Z_{\mathrm{K}}\right) \cdot \psi^{*}\left(\frac{-t}{n+\mathbb{E} Z_{\mathrm{K}}}\right)\right\} .
\end{gathered}
$$

The function $\psi^{*}(s):=((1+s) \log (1+s)-s) / 2$ for $s>-1$.

The proof of this result follows the same pattern as the argument from Theorem 3.1. In Section 4.5, we derive Theorem 4.1 as an immediate consequence.

4.1. Moment Generating Function of the Information Content. In addition to the variance, one may study other moments of the information content random variable. In particular, bounds for the moment generating function (mgf) of the centered information content lead to exponential tail bounds for the information content. Bobkov \& Madiman [BM11] proved the first result in this direction. More recently, Fradelizi et al. [FMW16] have obtained the optimal bound. 
Fact 4.2 (Information Content Mgf). Let $\mu: \mathbb{R}^{n} \rightarrow \mathbb{R}_{+}$be a log-concave probability density. For $\beta<1$,

$$
\mathbb{E} \mathrm{e}^{\beta\left(I_{\mu}-\mathbb{E} I_{\mu}\right)} \leq \mathrm{e}^{n \varphi(\beta)},
$$

where $\varphi(s):=-s-\log (1-s)$ for $s<1$. The information content random variable $I_{\mu}$ is defined in (3.1).

4.2. Information Content and Intrinsic Volumes. We extract concentration inequalities for the intrinsic volume random variable $Z_{\mathrm{K}}$ by studying its (centered) exponential moments. Define

$$
m_{\mathrm{K}}(\theta):=\mathbb{E} \mathrm{e}^{\theta\left(Z_{\mathrm{K}}-\mathbb{E} Z_{\mathrm{K}}\right)} \quad \text { for } \theta \in \mathbb{R} .
$$

The first step in the argument is to represent the mgf in terms of the information content random variable $H_{\mathrm{K}}$ defined in (3.5).

Proposition 4.3 (Mgf of Intrinsic Volume Random Variable). Let $\mathrm{K} \subset \mathbb{R}^{n}$ be a nonempty convex body with intrinsic volume random variable $Z_{\mathrm{K}}$ and information content random variable $H_{\mathrm{K}}$. For $\theta \in \mathbb{R}$,

$$
m_{\mathrm{K}}(\theta)=\mathrm{e}^{-\varphi(\beta) \mathbb{E} H_{\mathrm{K}}} \cdot \mathbb{E} \mathrm{e}^{\beta\left(H_{\mathrm{K}}-\mathbb{E} H_{\mathrm{K}}\right)} \quad \text { where } \beta:=1-\mathrm{e}^{2 \theta} .
$$

The function $\varphi$ is defined in Fact 4.2.

Proof. The formula (2.3) from Example 2.8 yields the identity

$$
\mathbb{E} \mathrm{e}^{\theta\left(Z_{\mathrm{K}}-n\right)}=\frac{1}{W(\mathrm{~K})} \int_{\mathbb{R}^{n}} \mathrm{e}^{\left(1-\mathrm{e}^{2 \theta}\right) \cdot \pi \operatorname{dist}^{2}(\boldsymbol{x}, \mathrm{K})} \cdot \mathrm{e}^{-\pi \operatorname{dist}^{2}(\boldsymbol{x}, \mathrm{K})} \mathrm{d} \boldsymbol{x}=\mathbb{E} \mathrm{e}^{\left(1-\mathrm{e}^{2 \theta}\right) H_{\mathrm{K}}} .
$$

We can transfer this result to obtain another representation for $m_{\mathrm{K}}$. First, use the identity (3.8) to replace $\mathbb{E} Z_{\mathrm{K}}$ with $\mathbb{E} H_{\mathrm{K}}$. Then invoke the last display to reach

$$
\begin{aligned}
m_{\mathrm{K}}(\theta)=\mathbb{E} \mathrm{e}^{\theta\left(Z_{\mathrm{K}}-\mathbb{E} Z_{\mathrm{K}}\right)} & =\mathrm{e}^{2 \theta \mathbb{E} H_{\mathrm{K}}} \mathbb{E} \mathrm{e}^{\theta\left(Z_{\mathrm{K}}-n\right)} \\
& =\mathrm{e}^{2 \theta \mathbb{E} H_{\mathrm{K}}} \mathbb{E} \mathrm{e}^{\left(1-\mathrm{e}^{2 \theta}\right) H_{\mathrm{K}}} \\
& =\mathrm{e}^{\left(1+2 \theta-\mathrm{e}^{2 \theta}\right) \mathbb{E} H_{\mathrm{K}}} \mathbb{E} \mathrm{e}^{\left(1-\mathrm{e}^{2 \theta}\right)\left(H_{\mathrm{K}}-\mathbb{E} H_{\mathrm{K}}\right)}=\mathrm{e}^{(\beta+\log (1-\beta)) \mathbb{E} H_{\mathrm{K}}} \mathbb{E} \mathrm{e}^{\beta\left(H_{\mathrm{K}}-\mathbb{E} H_{\mathrm{K}}\right)} .
\end{aligned}
$$

In the last step, we have made the change of variables $\beta=1-\mathrm{e}^{2 \theta}$. Finally, identify the value $-\varphi(\beta)$ in the first exponent.

4.3. A Bound for the Mgf. We are now prepared to bound the mgf $m_{\mathrm{K}}$. This result will lead directly to concentration of the intrinsic volume random variable.

Proposition 4.4 (A Bound for the Mgf). Let $\mathrm{K} \subset \mathbb{R}^{n}$ be a nonempty convex body with intrinsic volume random variable $Z_{\mathrm{K}}$. For $\theta \in \mathbb{R}$,

$$
m_{\mathrm{K}}(\theta) \leq \mathrm{e}^{\psi(\theta)\left(n+\mathbb{E} Z_{\mathrm{K}}\right)},
$$

where $\psi(s):=\left(\mathrm{e}^{2 s}-2 s-1\right) / 2$ for $s \in \mathbb{R}$.

Proof. For the parameter $\beta=1-\mathrm{e}^{2 \theta}$, Proposition 4.3 yields

$$
\begin{aligned}
m_{\mathrm{K}}(\theta) & =\mathrm{e}^{-\varphi(\beta) \mathbb{E} H_{\mathrm{K}}} \mathbb{E} \mathrm{e}^{\beta\left(H_{\mathrm{K}}-\mathbb{E} H_{\mathrm{K}}\right)} \\
& =\mathrm{e}^{-\varphi(\beta) \mathbb{E} H_{\mathrm{K}}} \mathbb{E} \mathrm{e}^{\beta\left(I_{\mu_{\mathrm{K}}}-\mathbb{E} I_{\mu_{\mathrm{K}}}\right)} \\
& \leq \mathrm{e}^{-\varphi(\beta) \mathbb{E} H_{\mathrm{K}}} \cdot \mathrm{e}^{n \varphi(\beta)} \\
& =\mathrm{e}^{-\varphi(\beta)\left(n-\mathbb{E} Z_{\mathrm{K}}\right) / 2} \cdot \mathrm{e}^{n \varphi(\beta)}=\mathrm{e}^{\varphi(\beta)\left(n+\mathbb{E} Z_{\mathrm{K}}\right) / 2} .
\end{aligned}
$$

To reach the second line, we use the equivalence (3.7) for the central moments. The inequality is Fact 4.2, the mgf bound for the information content $I_{\mu_{\mathrm{K}}}$ of the log-concave density $\mu_{\mathrm{K}}$. Afterward, we invoke (3.8) to pass from the information content random variable $H_{\mathrm{K}}$ to the intrinsic volume random variable $Z_{\mathrm{K}}$. The next step is algebraic. The result follows when we return from the variable $\beta$ to the variable $\theta$, leading to the appearance of the function $\psi$. 
4.4. Proof of Theorem 4.1. The Laplace transform method, combined with the mgf bound from Proposition 4.4, produces Bennett-type inequalities for the intrinsic volume random variable. In brief,

$$
\begin{aligned}
\mathbb{P}\left\{Z_{\mathrm{K}}-\mathbb{E} Z_{\mathrm{K}} \geq t\right\} & \leq \inf _{\theta>0} \mathrm{e}^{-\theta t} \cdot m_{\mathrm{K}}(\theta) \\
& \leq \inf _{\theta>0} \mathrm{e}^{-\theta t+\psi(\theta)\left(n+\mathbb{E} Z_{\mathrm{K}}\right)}=\exp \left\{-\left(n+\mathbb{E} Z_{\mathrm{K}}\right) \cdot \psi^{*}\left(\frac{t}{n+\mathbb{E} Z_{\mathrm{K}}}\right)\right\} .
\end{aligned}
$$

The Fenchel-Legendre conjugate $\psi^{*}$ of the function $\psi$ has the explicit form given in the statement of Theorem 4.1. The lower tail bound follows from the same argument.

4.5. Proof of Theorem 1.11. The concentration inequality in the main result, Theorem 4.1, follows when we weaken the inequalities obtained in the last section. Comparing derivatives, we can verify that $\psi^{*}(s) \geq\left(s^{2} / 4\right) /(1+s / 3)$ for all $s>-1$. For the interesting range, $0 \leq t \leq n$, we have

$$
\begin{array}{r}
\mathbb{P}\left\{Z_{\mathrm{K}}-\mathbb{E} Z_{\mathrm{K}} \geq t\right\} \leq \exp \left\{\frac{-t^{2} / 4}{n+\mathbb{E} Z_{\mathrm{K}}+t / 3}\right\} ; \\
\mathbb{P}\left\{Z_{\mathrm{K}}-\mathbb{E} Z_{\mathrm{K}} \leq-t\right\} \leq \exp \left\{\frac{-t^{2} / 4}{n+\mathbb{E} Z_{\mathrm{K}}-t / 3}\right\} .
\end{array}
$$

We may combine this pair of inequalities into a single bound:

$$
\mathbb{P}\left\{\left|Z_{\mathrm{K}}-\mathbb{E} Z_{\mathrm{K}}\right| \geq t\right\} \leq 2 \exp \left(\frac{-t^{2} / 4}{n+\mathbb{E} Z_{\mathrm{K}}+t / 3}\right) .
$$

Make the estimate $\mathbb{E} Z_{\mathrm{K}} \leq n$, and bound the denominator using $t \leq n$. This completes the argument.

\section{Example: Rectangular Parallelotopes}

In this section, we work out the intrinsic volume sequence of a rectangular parallelotope. This computation involves the generating function of the intrinsic volume sequence. Because of its elegance, we develop this method in more depth than we need to treat the example at hand.

5.1. Generating Functions and Intrinsic Volumes. To begin, we collect some useful information about the properties of the generating function of the intrinsic volumes.

Definition 5.1 (Intrinsic Volume Generating Function). The generating function of the intrinsic volumes of the convex body $\mathrm{K}$ is the polynomial

$$
G_{\mathrm{K}}(\lambda):=\sum_{j=0}^{n} \lambda^{j} V_{j}(\mathrm{~K})=W(\lambda \mathrm{K}) \text { for } \lambda>0 .
$$

We can use the generating function to read off some information about a convex body, including the total intrinsic volume and the central intrinsic volume. This is a standard result [Wil94, Sec. 4.1], so we omit the elementary argument.

Proposition 5.2 (Properties of the Generating Function). For each nonempty convex body $\mathrm{K} \subset \mathbb{R}^{n}$,

$$
W(\mathrm{~K})=G_{\mathrm{K}}(1) \quad \text { and } \quad \Delta(\mathrm{K})=\frac{G_{\mathrm{K}}^{\prime}(1)}{G_{\mathrm{K}}(1)}=\left(\log G_{\mathrm{K}}\right)^{\prime}(1) .
$$

As usual, the prime' denotes a derivative.

It is usually challenging to compute the intrinsic volumes of a convex body, but the following fact allows us to make short work of some examples.

Fact 5.3 (Direct Products). Let $C \subset \mathbb{R}^{n_{1}}$ and $\mathrm{K} \subset \mathbb{R}^{n_{2}}$ be nonempty convex bodies. The generating function of the intrinsic volumes of the convex body $\mathrm{C} \times \mathrm{K} \subset \mathbb{R}^{n_{1}+n_{2}}$ takes the form

$$
G_{\mathrm{C} \times \mathrm{K}}(\lambda)=G_{\mathrm{C}}(\lambda) \cdot G_{\mathrm{K}}(\lambda) \text {. }
$$

For completeness, we include a short proof inspired by Hadwiger [Had75]; see [SWo8, Lem. 14.2.1]. 
Proof. Abbreviate $n:=n_{1}+n_{2}$. For a point $\boldsymbol{x} \in \mathbb{R}^{n}$, write $\boldsymbol{x}=\left(\boldsymbol{x}_{1}, \boldsymbol{x}_{2}\right)$ where $\boldsymbol{x}_{i} \in \mathbb{R}^{n_{i}}$. Then

$$
\operatorname{dist}^{2}(\boldsymbol{x}, \mathrm{C} \times \mathrm{K})=\operatorname{dist}^{2}\left(\boldsymbol{x}_{1}, \mathrm{C}\right)+\operatorname{dist}^{2}\left(\boldsymbol{x}_{2}, \mathrm{~K}\right) .
$$

Invoke the formula (2.2) from Example 2.8 for the generating function of the intrinsic volumes (three times!). For $\lambda>0$,

$$
\begin{aligned}
\lambda^{-n} \sum_{j=0}^{n} \lambda^{j} V_{j}(\mathrm{C} \times \mathrm{K}) & =\int_{\mathbb{R}^{n}} \mathrm{e}^{-\lambda^{2} \pi \operatorname{dist}^{2}(\boldsymbol{x}, \mathrm{C} \times \mathrm{K})} \mathrm{d} \boldsymbol{x} \\
& =\int_{\mathbb{R}^{n_{1}}} \int_{\mathbb{R}^{n_{2}}} \mathrm{e}^{-\lambda^{2} \pi \operatorname{dist}^{2}\left(\boldsymbol{x}_{1}, \mathrm{C}\right)} \cdot \mathrm{e}^{-\lambda^{2} \pi \operatorname{dist}^{2}\left(\boldsymbol{x}_{2}, \mathrm{~K}\right)} \mathrm{d} \boldsymbol{x}_{1} \mathrm{~d} \boldsymbol{x}_{2} \\
& =\left(\lambda^{-n_{1}} \sum_{j=0}^{n_{1}} \lambda^{j} V_{j}(\mathrm{C})\right)\left(\lambda^{-n_{2}} \sum_{j=0}^{n_{2}} \lambda^{j} V_{j}(\mathrm{~K})\right) .
\end{aligned}
$$

Cancel the leading factors of $\lambda$ to complete the argument.

As a corollary, we can derive an expression for the central intrinsic volume of a direct product.

Corollary 5.4 (Central Intrinsic Volume of a Product). Let $C \subset \mathbb{R}^{n_{1}}$ and $\mathrm{K} \subset \mathbb{R}^{n_{2}}$ be nonempty convex bodies. Then

$$
\Delta(\mathrm{C} \times \mathrm{K})=\Delta(\mathrm{C})+\Delta(\mathrm{K}) .
$$

Proof. According to Proposition 5.2 and Fact 5.3,

$$
\begin{aligned}
\Delta(\mathrm{C} \times \mathrm{K}) & =\left(\log G_{\mathrm{C} \times \mathrm{K}}\right)^{\prime}(1)=\left(\log \left(G_{\mathrm{C}} G_{\mathrm{K}}\right)\right)^{\prime}(1) \\
& =\left(\log G_{\mathrm{C}}+\log G_{\mathrm{K}}\right)^{\prime}(1)=\left(\log G_{\mathrm{C}}\right)^{\prime}(1)+\left(\log G_{\mathrm{K}}\right)^{\prime}(1)=\Delta(\mathrm{C})+\Delta(\mathrm{K}) .
\end{aligned}
$$

This is what we needed to show.

5.2. Intrinsic Volumes of a Rectangular Parallelotope. Using Fact 5.3, we quickly compute the intrinsic volumes and related statistics for a rectangular parallelotope.

Proposition 5.5 (Rectangular Parallelotopes). For parameters $s_{1}, s_{2}, \ldots, s_{n} \geq 0$, construct the rectangular parallelotope

$$
\mathrm{P}:=\left[0, s_{1}\right] \times\left[0, s_{2}\right] \times \cdots \times\left[0, s_{n}\right] \subset \mathbb{R}^{n} .
$$

The generating function for the intrinsic volumes of the parallelotope $\mathrm{P}$ satisfies

$$
G_{\mathrm{P}}(\lambda)=\prod_{i=1}^{n}\left(1+\lambda s_{i}\right) .
$$

In particular, $V_{j}(\mathrm{~K})=e_{j}\left(s_{1}, \ldots, s_{n}\right)$, where $e_{j}$ denotes the $j$ th elementary symmetric function. The total intrinsic volume and central intrinsic volume satisfy

$$
W(\mathrm{P})=\prod_{i=1}^{n}\left(1+s_{i}\right) \text { and } \Delta(\mathrm{P})=\sum_{i=1}^{n} \frac{s_{i}}{1+s_{i}} .
$$

Proof. Let $s \geq 0$. By direct calculation from Definition 1.1, the intrinsic volumes of the interval $[0, s] \subset \mathbb{R}^{1}$ are $V_{0}([0, s])=1$ and $V_{1}([0, s])=s$. Thus,

$$
G_{[0, s]}(\lambda)=\sum_{j=0}^{1} \lambda^{j} V_{j}([0, s])=1+\lambda s .
$$

Fact 5.3 implies that the generating function for the intrinsic volumes of the parallelotope $P$ is

$$
G_{\mathrm{P}}(\lambda):=\sum_{j=0}^{n} \lambda^{j} V_{j}(\mathrm{P})=\prod_{i=0}^{n}\left(1+\lambda s_{i}\right) .
$$

We immediately obtain formulas for the total intrinsic volume and the central intrinsic volume from Proposition 5.2. Alternatively, we can compute the central intrinsic volume of an interval $[0, s]$ and use Corollary 5.4 to extend this result to the parallelotope P. 
5.3. Intrinsic Volumes of a Cube. As an immediate consequence of Proposition 5.5, we obtain a clean result on the intrinsic volumes of a scaled cube.

Corollary 5.6 (Cubes). Let $\mathrm{Q}_{n} \subset \mathbb{R}^{n}$ be the unit cube. For $s \geq 0$, the normalized intrinsic volumes of the scaled cube $s Q_{n}$ coincide with a binomial distribution. For each $j=0,1,2, \ldots, n$,

$$
\tilde{V}_{j}\left(s \mathrm{Q}_{n}\right)=\left(\begin{array}{c}
n \\
j
\end{array}\right) \cdot p^{j}(1-p)^{n-j} \quad \text { where } \quad p=\frac{s}{1+s} .
$$

In particular, the central intrinsic volume of the scaled cube is

$$
\Delta\left(s \mathrm{Q}_{n}\right)=n p=\frac{n s}{1+s} .
$$

Corollary 5.6 plays a starring role in our analysis of the intrinsic volume sequences that attain the maximum entropy.

We can also use Corollary 5.6 to test our results on the variance and concentration properties of the intrinsic volume sequence by comparing them with exact computations for the cube. Fix a number $s \geq 0$, and let $p=s /(1+s)$. Then

$$
\operatorname{Var}\left[Z_{s Q_{n}}\right]=n p(1-p)=\frac{n s}{(1+s)^{2}} .
$$

Meanwhile, Theorem 3.1 gives the upper bound

$$
\operatorname{Var}\left[Z_{s \mathrm{Q}_{n}}\right] \leq 2(n+n p)=\frac{2 n(1+2 s)}{1+s} .
$$

For $s=1$, the ratio of the upper bound to the exact variance is 12 . For $s \approx 0$ and $s \rightarrow \infty$, the ratio becomes arbitrarily large. Similarly, Theorem 4.1 gives a qualitatively good description for $s=1$, but its predictions are far less accurate for small and large $s$. There remains more work to do!

\section{Maximum-Entropy Distributions of Intrinsic Volumes}

We have been using probabilistic methods to study the intrinsic volumes of a convex body, and we have seen that the intrinsic volume sequence is concentrated, as reflected in the variance bound (Theorem 3.1) and the exponential tail bounds (Theorem 4.1). Therefore, it is natural to consider other measures of the dispersion of the sequence. We recall Definition 1.12, of the intrinsic entropy, which is the entropy of the normalized intrinsic volume sequence. This concept turns out to be interesting.

In this section, we will establish Theorem 1.13. This result states that, among all convex bodies with a fixed central intrinsic volume, a scaled cube has the largest entropy. Moreover, the unit-volume cube has the largest intrinsic entropy among all convex bodies in a fixed dimension. We prove this theorem using some recent observations from information theory.

6.1. Ultra-Log-Concavity and Convex Bodies. The key step in proving Theorem 1.13 is to draw a connection between intrinsic volumes and ultra-log-concave sequences. We begin with an important definition.

Definition 6.1 (Ultra-Log-Concave Sequence). A nonnegative sequence $\left\{a_{j}: j=0,1,2, \ldots\right\}$ is called ultra-log-concave, briefly $U L C$, if it satisfies the relations

$$
j \cdot a_{j}^{2} \geq(j+1) \cdot a_{j+1} a_{j-1} \text { for } j=1,2,3, \ldots
$$

It is equivalent to say that the sequence $\left\{j ! a_{j}: j=0,1,2, \ldots\right\}$ is log-concave.

Among all finitely supported ULC probability distributions, the binomial distributions have the maximum entropy. This result was obtained by Yaming Yu [Yuo8] using methods developed by Oliver Johnson [Joho7] for studying the maximum-entropy properties of Poisson distributions. 
Fact 6.2 (Binomial Distributions Maximize Entropy). Let $p \in[0,1]$, and fix a natural number $n$. Among all ULC probability distributions with mean pn that are supported on $\{0,1,2, \ldots, n\}$, the binomial distribution $\operatorname{BIN}(p, n)$ has the maximum entropy.

These facts are relevant to our discussion because the intrinsic volumes of a convex body form an ultra-log-concave sequence.

Fact 6.3 (Intrinsic Volumes are ULC). The normalized intrinsic volumes of a nonempty convex body in $\mathbb{R}^{n}$ compose a ULC probability distribution supported on $\{0,1,2, \ldots, n\}$.

This statement is a consequence of the Alexandrov-Fenchel inequalities [Sch14, Sec. 7.3]; see the papers of Chevet [Che76] and McMullen [McM91].

6.2. Proof of Theorem 1.13. With this information at hand, we quickly establish the main result of the section. Recall that $\mathrm{Q}_{n}$ denotes the unit-volume cube in $\mathbb{R}^{n}$. Let $\mathrm{K} \subset \mathbb{R}^{n}$ be a nonempty convex body. Define the number $p \in[0,1)$ by the relation $p n=\Delta(\mathrm{K})$. According to Corollary 5.6, the scaled cube $s \mathrm{Q}_{n}$ satisfies

$$
\Delta\left(s \mathrm{Q}_{n}\right)=p n=\Delta(\mathrm{K}) \text { when } \quad s=\frac{p}{1-p} .
$$

Fact 6.3 ensures that the normalized intrinsic volume sequence of the convex body $K$ is a ULC probability distribution supported on $\{0,1,2, \ldots, n\}$. Since $\mathbb{E} Z_{\mathrm{K}}=\Delta(\mathrm{K})=p n$, Fact 6.2 now delivers

$$
\operatorname{IntEnt}(\mathrm{K})=\operatorname{Ent}\left[Z_{\mathrm{K}}\right] \leq \operatorname{Ent}[\operatorname{BiN}(p, n)]=\operatorname{Ent}\left[Z_{s \mathbf{Q}_{n}}\right]=\operatorname{IntEnt}\left(s \mathrm{Q}_{n}\right) .
$$

We have used Corollary 5.6 again to see that $Z_{s Q_{n}} \sim \operatorname{BIN}(p, n)$. The remaining identities are simply the definition of the intrinsic entropy. In other words, the scaled cube has the maximum intrinsic entropy among all convex bodies that share the same central intrinsic volume.

It remains to show that the unit-volume cube has maximum intrinsic entropy among all convex bodies. Continuing the analysis in the last display, we find that

$$
\operatorname{IntEnt}(\mathrm{K}) \leq \operatorname{Ent}[\operatorname{Bin}(p, n)] \leq \operatorname{Ent}[\operatorname{Bin}(1 / 2, n)]=\operatorname{Ent}\left[Z_{\mathrm{Q}_{n}}\right]=\operatorname{IntEnt}\left(\mathrm{Q}_{n}\right) .
$$

Indeed, among the binomial distributions $\operatorname{BIN}(p, n)$ for $p \in[0,1]$, the maximum entropy distribution is $\operatorname{BIN}(1 / 2, n)$. But this is the distribution of $Z_{Q_{n}}$, the intrinsic volume random variable of the unit cube $\mathrm{Q}_{n}$. This observation implies the remaining claim in Theorem 1.13.

\section{ACKNOWLEDGMENTS AND AfFiliations}

We are grateful to Emmanuel Milman for directing us to the literature on concentration of information. Dennis Amelunxen, Sergey Bobkov, and Michel Ledoux also gave feedback at an early stage of this project. Ramon Van Handel provided valuable comments and citations, including the fact that ULC sequences concentrate. We thank the anonymous referee for a careful reading and constructive remarks.

Parts of this research were completed at Luxembourg University and at the Institute for Mathematics and its Applications (IMA) at the University of Minnesota. Giovanni Peccati is supported by the internal research project STARS (R-AGR-0502-10) at Luxembourg University. Joel A. Tropp gratefully acknowledges support from ONR award Nooo14-11-1002 and the Gordon \& Betty Moore Foundation.

Martin Lotz (martin. lotz@warwick.ac.uk) is affiliated with the Mathematics Institute, University of Warwick. Michael McCoy (mike.mccoy@getcruise.com) is with Cruise Automation. Ivan Nourdin (ivan.nourdin@uni.lu) and Giovanni Peccati (giovanni.peccati@gmail.com) are with the Unité de Recherche en Mathématiques, University of Luxembourg. Joel A. Tropp (jtropp@cms.caltech.edu) is with the Department of Computing \& Mathematical Sciences, California Institute of Technology. 


\section{REFERENCES}

[AAGM15] S. Artstein-Avidan, A. Giannopoulos, and V. D. Milman. Asymptotic geometric analysis. Part I, volume 202 of Mathematical Surveys and Monographs. American Mathematical Society, Providence, RI, 2015.

[ALMT14] D. Amelunxen, M. Lotz, M. B. McCoy, and J. A. Tropp. Living on the edge: phase transitions in convex programs with random data. Inf. Inference, 3(3):224-294, 2014.

[AS16] K. Adiprasito and R. Sanyal. Whitney numbers of arrangements via measure concentration of intrinsic volumes. Available at http://arXiv.org/abs/1606.09412, June 2016.

[ATo7] R. J. Adler and J. E. Taylor. Random fields and geometry. Springer Monographs in Mathematics. Springer, New York, 2007.

[Bal91] K. Ball. Volume ratios and a reverse isoperimetric inequality. J. London Math. Soc. (2), 44(2):351-359, 1991.

[Bal97] K. Ball. An elementary introduction to modern convex geometry. In Flavors of geometry, volume 31 of Math. Sci. Res. Inst. Publ., pages 1-58. Cambridge Univ. Press, Cambridge, 1997.

[BL76] H. J. Brascamp and E. H. Lieb. On extensions of the Brunn-Minkowski and Prékopa-Leindler theorems, including inequalities for $\log$ concave functions, and with an application to the diffusion equation. J. Functional Analysis, 22(4):366-389, 1976.

[BM11] S. Bobkov and M. Madiman. Concentration of the information in data with log-concave distributions. Ann. Probab., 39(4):1528-1543, 2011.

[CDPPo9] P. Caputo, P. Dai Pra, and G. Posta. Convex entropy decay via the Bochner-Bakry-Emery approach. Ann. Inst. Henri Poincaré Probab. Stat., 45(3):734-753, 2009.

[Che76] S. Chevet. Processus Gaussiens et volumes mixtes. Z. Wahrscheinlichkeitstheorie und Verw. Gebiete, 36(1):47-65, 1976.

[Fed59] H. Federer. Curvature measures. Trans. Amer. Math. Soc., 93:418-491, 1959.

[FMW16] M. Fradelizi, M. Madiman, and L. Wang. Optimal concentration of information content for log-concave densities. In High dimensional probability VII, volume 71 of Progr. Probab., pages 45-6o. Springer, 2016.

[GNP17] L. Goldstein, I. Nourdin, and G. Peccati. Gaussian phase transitions and conic intrinsic volumes: Steining the Steiner formula. Ann. Appl. Probab., 27(1):1-47, 2017.

[Gruo7] P. M. Gruber. Convex and discrete geometry, volume 336 of Grundlehren der Mathematischen Wissenschaften [Fundamental Principles of Mathematical Sciences]. Springer, Berlin, 2007.

[Had51] H. Hadwiger. Beweis eines Funktionalsatzes für konvexe Körper. Abh. Math. Sem. Univ. Hamburg, 17:69-76, 1951.

[Had52] H. Hadwiger. Additive Funktionale $k$-dimensionaler Eikörper. I. Arch. Math., 3:470-478, 1952.

[Had57] H. Hadwiger. Vorlesungen über Inhalt, Oberfläche und Isoperimetrie. Springer-Verlag, Berlin-Göttingen-Heidelberg, 1957.

[Had75] H. Hadwiger. Das Wills'sche Funktional. Monatsh. Math., 79:213-221, 1975.

[Joho7] O. Johnson. Log-concavity and the maximum entropy property of the Poisson distribution. Stochastic Process. Appl., 117(6):791-802, 2007.

[KR97] D. A. Klain and G.-C. Rota. Introduction to geometric probability. Lezioni Lincee. [Lincei Lectures]. Cambridge University Press, Cambridge, 1997.

[McC13] M. B. McCoy. A geometric analysis of convex demixing. ProQuest LLC, Ann Arbor, MI, 2013. Thesis (Ph.D.)California Institute of Technology.

[McM75] P. McMullen. Non-linear angle-sum relations for polyhedral cones and polytopes. Math. Proc. Cambridge Philos. Soc., 78(2):247-261, 1975.

[McM91] P. McMullen. Inequalities between intrinsic volumes. Monatsh. Math., 111(1):47-53, 1991.

[MT14a] M. B. McCoy and J. A. Tropp. From Steiner formulas for cones to concentration of intrinsic volumes. Discrete Comput. Geom., 51(4):926-963, 2014.

[MT14b] M. B. McCoy and J. A. Tropp. Sharp recovery bounds for convex demixing, with applications. Found. Comput. Math., 14(3):503-567, 2014.

[MT17] M. B. McCoy and J. A. Tropp. The achievable performance of convex demixing. ACM Report 2017-02, California Institute of Technology, 2017. Manuscript dated 28 Sep. 2013.

[Ngu13] V. H. Nguyen. Inégalités Fonctionelles et Convexité. PhD thesis, Université Pierrre et Marie Curie (Paris VI), 2013.

[Pis89] G. Pisier. The volume of convex bodies and Banach space geometry, volume 94 of Cambridge Tracts in Mathematics. Cambridge University Press, Cambridge, 1989.

[PPV17] G. Paouris, P. Pivovarov, and P. Valettas. On a quantitative reversal of Alexandrov's inequality. Available at http://arxiv.org/abs/1702.05762, Feb. 2017.

[Sano4] L. A. Santaló. Integral geometry and geometric probability. Cambridge Mathematical Library. Cambridge University Press, Cambridge, second edition, 2004. With a foreword by Mark Kac.

[Sch14] R. Schneider. Convex bodies: the Brunn-Minkowski theory, volume 151 of Encyclopedia of Mathematics and its Applications. Cambridge University Press, Cambridge, expanded edition, 2014. 
[SH18] Y. Shenfeld and R. V. Handel. Mixed volumes and the Bochner method. Available at http://arXiv.org/abs/1811.08710, Nov. 2018.

[SWo8] R. Schneider and W. Weil. Stochastic and integral geometry. Probability and its Applications (New York). SpringerVerlag, Berlin, 2008.

[SW14] A. Saumard and J. A. Wellner. Log-concavity and strong log-concavity: a review. Stat. Surv., 8:45-114, 2014.

[Vit96] R. A. Vitale. The Wills functional and Gaussian processes. Ann. Probab., 24(4):2172-2178, 1996.

[Wan14] L. Wang. Heat Capacity Bound, Energy Fluctuations and Convexity. ProQuest LLC, Ann Arbor, MI, 2014. Thesis (Ph.D.)-Yale University.

[Wil73] J. M. Wills. Zur Gitterpunktanzahl konvexer Mengen. Elem. Math., 28:57-63, 1973.

[Wil94] H. S. Wilf. generatingfunctionology. Academic Press, Inc., Boston, MA, second edition, 1994.

[Yuo8] Y. Yu. On the maximum entropy properties of the binomial distribution. IEEE Trans. Inform. Theory, 54(7):3351$3353,2008$. 